\title{
BMJ Open The COMET (Comparison of Operative versus Monitoring and Endocrine Therapy) trial: a phase III randomised controlled clinical trial for low-risk ductal carcinoma in situ (DCIS)
}

\author{
E Shelley Hwang, ${ }^{1}$ Terry Hyslop, ${ }^{2}$ Thomas Lynch, ${ }^{1}$ Elizabeth Frank, ${ }^{3}$ Donna Pinto, ${ }^{3}$ \\ Desiree Basila, ${ }^{3}$ Deborah Collyar, ${ }^{3}$ Antonia Bennett, ${ }^{4}$ Celia Kaplan, ${ }^{5}$ \\ Shoshana Rosenberg, ${ }^{6}$ Alastair Thompson, ${ }^{7}$ Anna Weiss, ${ }^{8}$ Ann Partridge ${ }^{6}$
}

To cite: Hwang ES, Hyslop T, Lynch T, et al. The COMET (Comparison of Operative versus Monitoring and Endocrine Therapy) trial: a phase III randomised controlled clinical trial for low-risk ductal carcinoma in situ (DCIS). BMJ Open 2019;9:e026797. doi:10.1136/ bmjopen-2018-026797

- Prepublication history and additional material for this paper are available online. To view these files, please visit the journal online (http://dx.doi org/10.1136/bmjopen-2018026797).

Received 25 September 2018 Revised 5 November 2018 Accepted 6 November 2018
Check for updates

(C) Author(s) (or their employer(s)) 2019. Re-use permitted under CC BY-NC. No commercial re-use. See rights and permissions. Published by BMJ.

For numbered affiliations see end of article.

Correspondence to

Dr Thomas Lynch;

thomas.lynch2@duke.edu

\section{ABSTRACT}

Introduction Ductal carcinoma in situ (DCIS) is a noninvasive non-obligate precursor of invasive breast cancer. With guideline concordant care (GCC), DCIS outcomes are at least as favourable as some other early stage cancer types such as prostate cancer, for which active surveillance (AS) is a standard of care option. However, AS has not yet been tested in relation to DCIS. The goal of the COMET (Comparison of Operative versus Monitoring and Endocrine Therapy) trial for low-risk DCIS is to gather evidence to help future patients consider the range of treatment choices for low-risk DCIS, from standard therapies to AS. The trial will determine whether there may be some women who do not substantially benefit from current GCC and who could thus be safely managed with AS. This protocol is version 5 (11 July 2018). Any future protocol amendments will be submitted to Quorum Centralised Institutional Review Board/local institutional review boards for approval via the sponsor of the study (Alliance Foundation Trials).

Methods and analysis COMET is a phase III, randomised controlled clinical trial for patients with low-risk DCIS. The primary outcome is ipsilateral invasive breast cancer rate in women undergoing GCC compared with AS. Secondary objectives will be to compare surgical, oncological and patient-reported outcomes. Patients randomised to the GCC group will undergo surgery as well as radiotherapy when appropriate; those in the AS group will be monitored closely with surgery only on identification of invasive breast cancer. Patients in both the GCC and AS groups will have the option of endocrine therapy. The total planned accrual goal is 1200 patients.

Ethics and dissemination The COMET trial will be subject to biannual formal review at the Alliance Foundation Data Safety Monitoring Board meetings. Interim analyses for futility/safety will be completed annually, with reporting following Consolidated Standards of Reporting Trials (CONSORT) guidelines for noninferiority trials.

Trial registration number NCT02926911; Pre-results.
Strengths and limitations of this study

- Comparison of Operative versus Monitoring and Endocrine Therapy (COMET) is a phase III randomised controlled clinical trial.

- Ongoing data collected from women who decline randomisation will provide valuable information about the potential for selection bias/enable the study to be made more generalisable.

- The comparator arms are very different from each other.

- There exists considerable variation between pathologists in the diagnosis of ductal carcinoma in situ.

\section{INTRODUCTION}

Ductal carcinoma in situ (DCIS): potential risks and burdens

Annually, approximately 65 million women undergo mammographic screening in the USA at a cost of over 13 billion dollars. Almost one in 1300 mammograms (MMGs) will detect ductal carcinoma in situ or DCIS, ${ }^{1}$ with more than 50000 women in the USA alone diagnosed with DCIS each year. Almost all diagnoses are made in completely asymptomatic individuals. ${ }^{2}$ Without treatment, it is estimated that only $20 \%-30 \%$ of DCIS will progress to invasive breast cancer. ${ }^{34}$ However, once diagnosed, over $97 \%$ are treated according to current guidelines with a combination of surgery, radiation and endocrine therapy-treatments similar to those recommended for patients with invasive breast cancer.

The term 'overdiagnosis' has been used to define conditions that look like early cancer, but are not destined to cause symptoms or death. ${ }^{5}$ In 2013, an independent review commissioned by the Department of Health 
in the UK established that screening saves lives but also that overdiagnosis exists. ${ }^{6}$ There is a general consensus that much of the overdiagnosis and overtreatment burden in breast cancer derives from the treatment of DCIS. Currently, almost all DCIS is treated according to guideline concordant care (GCC); of those treated for low-risk DCIS, some patients will not benefit if they never develop invasive breast cancer. One possible approach to GCC for low-risk lesions is active surveillance (AS). Currently, only $3 \%$ of women in the USA with DCIS opt for AS. Given that much of the treatment for low-risk DCIS may represent overtreatment, there has been global interest to address whether AS, with intervention only for invasive breast cancer, would be sufficient for those women unlikely to have a future DCIS or invasive breast cancer.

\section{Current gaps in evidence}

Current treatment options routinely offered for DCIS include surgery (lumpectomy or mastectomy), radiation and endocrine therapy. These options constitute GCC according to National Comprehensive Cancer Network treatment recommendations. ${ }^{7}$ Between 1991 and $2010,23.8 \%$ of women diagnosed with DCIS in the USA underwent unilateral mastectomy $(4.5 \%$ bilateral mastectomy), $43 \%$ lumpectomy with radiation and $26.5 \%$ lumpectomy without radiation. ${ }^{8}$ Published UK screening data suggest that in some cases, major surgical 'cancer' treatment of low-risk DCIS is unnecessary, inappropriate and misleading for the recipient. ${ }^{9}$ In those women who undergo surgical treatment for DCIS, there may be both short-term and long-term morbidities, including poor cosmesis and the risk of developing persistent pain at the surgical site, with estimates ranging from $25 \%$ to $68 \% .{ }^{10-13}$ In addition, patients may experience complications from radiation (cardiac or pulmonary symptoms, secondary malignancies) or reconstruction (infection, loss of implant, need for multiple surgeries). To date, among the $97 \%$ of women with DCIS treated with GCC, neither randomised trials nor observational studies have shown a survival advantage of any one treatment option over another. ${ }^{14}$ Moreover, none of the treatments has ever been compared in a rigorous fashion to AS. The COMET (Comparison of Operative versus Monitoring and Endocrine Therapy) trial for low-risk DCIS is a 5-year phase III, randomised controlled clinical trial that commenced on 1 July 2016. The study was designed with a specific objective: to determine the risks and benefits of GCC compared with those of AS for low-risk DCIS. This protocol is based on version 5 (dated 11 July 2018), approved by QUORUM Centralised Institutional Review Board (CIRB) and all local institutional review boards (IRBs) where relevant. Any future protocol amendments will be submitted to Quorum CIRB or local IRBs, in accordance with institutional requirements, via the sponsor of the study (Alliance Foundation Trials).
METHODS AND ANALYSIS

\section{Trial design and setting}

COMET is a phase III randomised controlled clinical trial for low-risk DCIS (figure 1) with two comparator arms, GCC and AS. The study, funded by the Patient-Centered Outcomes Research Institute, is conducted through the Alliance for Clinical Trials in Oncology cooperative group network with plans to open at up to 100 sites in the USA (a list of currently activated sites can be found at ClinicalTrials.gov-NCT02926911). Patients with a new diagnosis of DCIS are identified at participating Alliance study sites and screened for eligibility. Written informed consent is obtained prior to randomisation by site staff, including consent for the potential use of biological specimens in future studies (online supplementary appendix 1). Alliance has obtained a Certificate of Confidentiality from the Department of Health and Human Services in order to protect the privacy of individuals who are subjects of research by withholding their names and other identifying characteristics from all persons not connected with the conduct of Alliance research.

Data collection activities are embedded within the Alliance Statistics and Data Center infrastructure. Resource and data management for the trial follow the established Alliance standard operating processes for the collection, storage and analysis of online case report forms and other data. These procedures include all quality assurance processes that are in place for Alliance clinical trials as well as the use of Medidata Rave as the electronic data capture tool.

\section{Eligibility criteria}

Inclusion criteria for COMET were designed to select a group of English-speaking and Spanish-speaking patients at low risk for invasive breast cancer progression based on retrospective epidemiological data. Low-risk criteria were identified for clinical, radiological and pathological features. As a pragmatic trial, central review of imaging and pathology is not performed in real time, but reviewed post hoc. However, given the known limited inter-reviewer correlation between pathologists in the diagnosis of DCIS, the inclusion criteria require that at least two pathologists deem that the histological features meet COMET pathology eligibility criteria. A complete list of COMET inclusion and exclusion criteria are presented in table 1.

\section{Allocation and randomisation}

Allocation and randomisation is conducted by Alliance site staff. Randomisation is computer-generated via Medidata Rave and stratified based on the following factors: age at diagnosis: <55, 55-65, >65; maximum diameter of microcalcifications: $<2 \mathrm{~cm}, 2-5 \mathrm{~cm},>5 \mathrm{~cm}$ and DCIS nuclear grade: I or II. We record whether the patient has had prior surgical excision for the index diagnosis; this variable will be used for subset analysis but will not be a stratification factor. 


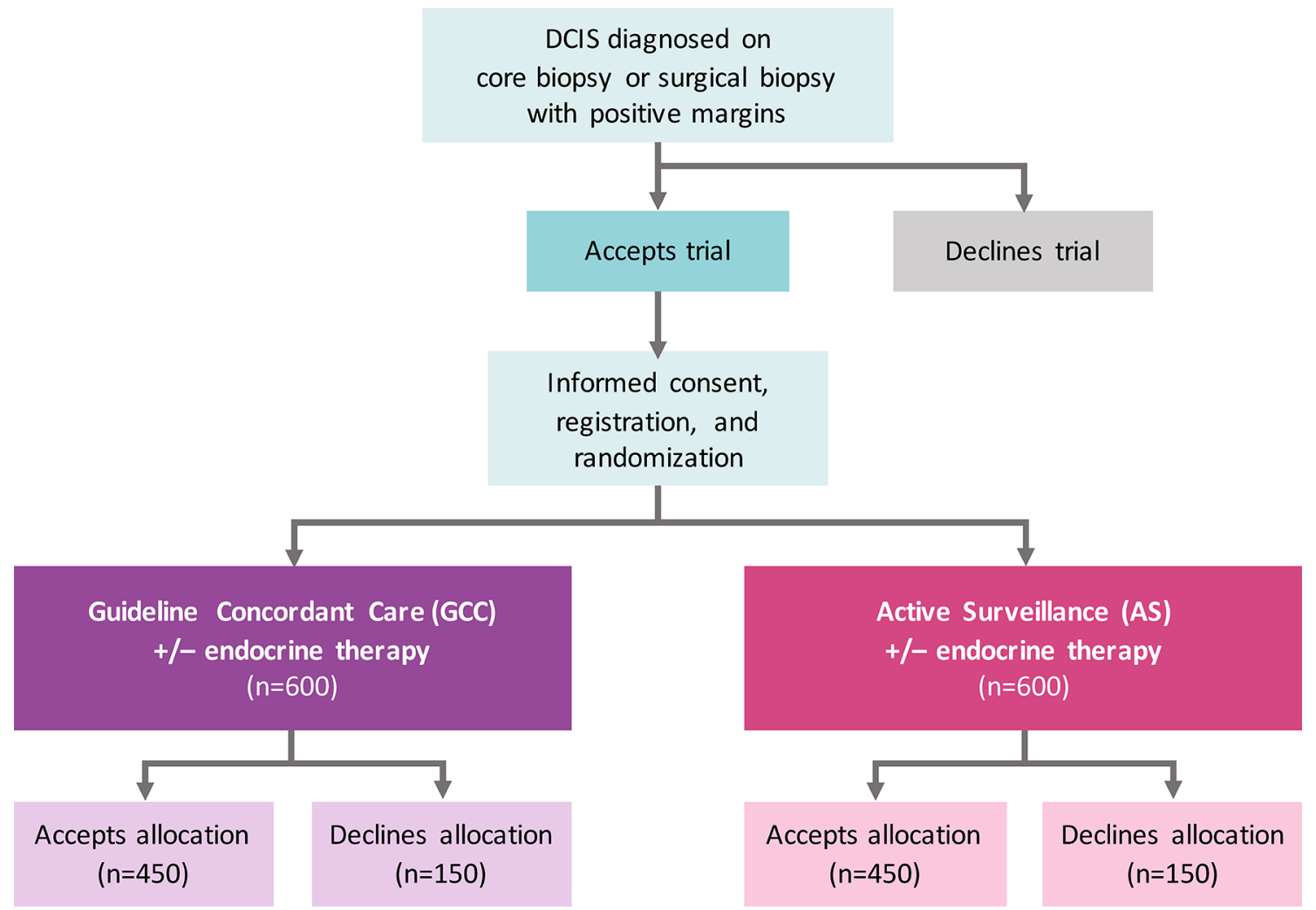

Figure 1 COMET trial schema. Patient flow for accrual and registration. Eligibility criteria for low-risk DCIS include 40 years of age or older, grade I/II DCIS without invasive breast cancer diagnosed on core, vacuum-assisted or surgical biopsy; ER(+) and/ or PR(+); HER2(-); and no mass on physical examination or imaging with exception of fibroadenoma at a distinct/separate site from the site of DCIS. The primary study endpoint on which the sample size is based is rate of 2-year invasive breast cancer diagnosis among patients randomised to GCC compared with AS. ITT analyses adjusted for drop-out, non-compliance and contamination will be performed on all randomised patients including those who do and do not accept the arm to which they are randomised. Patient-reported outcome surveys will be collected from all patients who are registered for the study, including those who crossover. Mammograms will be performed q6 months for the index breast and q12 months for the contralateral breast in the AS arm and q12 months in both the index and contralateral breast in the GCC arm. No chest wall imaging will be performed if mastectomy has been performed. AS, active surveillance; COMET, Comparison of Operative versus Monitoring and Endocrine Therapy; DCIS, ductal carcinoma in situ; ER(+), oestrogen receptor positive; GCC, guideline concordant care; HER2 $(-)$, human epidermal growth factor 2 negative; ITT, intention to treat; PR(+), progesterone receptor positive; q, every.

Use of endocrine therapy is permitted in both arms with adherence and duration of therapy recorded. Although women are not recruited to the COMET trial if they do not agree to randomisation, those women who are consented but then decline randomisation (or their allocated arm) are still eligible to continue participation in the study if they agree to provide follow-up and survey data. The demographics of the cohort declining their allocated arm will be compared with those of women who adhere to the randomisation. It is anticipated that these data will provide valuable information about the potential for selection bias and will ultimately enable the study to be made more generalisable.

Both intent-to-treat and per-protocol analyses can be biased in the presence of drop-out and non-compliance. ${ }^{1516}$ Thus, we intend to complete both of these analyses as sensitivity analyses, but the primary analysis approach will be based on an estimate of the treatment effect among those who comply with arm allocation. ${ }^{17}$

\section{STUDY ARMS}

\section{Guideline concordant care}

Surgery

Patients randomised to the GCC arm will undergo appropriate surgery for DCIS according to local guidelines. It is expected that patients will complete definitive surgery within 60 days of randomisation. Data on all related surgical procedures, including data on immediate or delayed breast reconstruction, will be collected. If a patient randomised to the GCC arm opts for AS, they will be considered as a 'crossover' and will continue to participate in completion of patient-reported outcome surveys.

\section{Radiotherapy}

The recommendation for post-surgical radiotherapy should be decided following surgery and recommended according to standard local protocols. The use of post-surgical radiotherapy is not mandated within the trial. However, data pertaining to the use of radiotherapy will be collected. 
Table 1 Eligibility criteria for the COMET trial

\begin{tabular}{|c|c|}
\hline Inclusion criteria & Exclusion criteria \\
\hline $\begin{array}{l}\text { New diagnosis of DCIS without invasive breast cancer } \\
\text { Unilateral, bilateral, unifocal, or multifocal DCIS } \\
\text { A patient who has had a lumpectomy with positive margins as part of } \\
\text { their treatment for a current DCIS diagnosis is eligible } \\
\text { No previous history of breast cancer (DCIS or invasive cancer) in } \\
\text { either breast prior to current DCIS diagnosis } \\
\text { 40years of age or older at the time of DCIS diagnosis } \\
\text { ECOG performance status } 0 \text { or } 1 \\
\text { No contraindication for surgery } \\
\text { Baseline imaging: } \\
\text { - Unilateral DCIS: contralateral normal mammogram } \leq 6 \text { months of } \\
\text { registration and ipsilateral breast imaging } \leq 120 \text { days of registration } \\
\text { - Bilateral DCIS: bilateral breast imaging } \leq 120 \text { days of registration } \\
\text { Pathological criteria: } \\
\text { - ADH suspicious for DCIS } \\
\text { - Any grade I or grade II DCIS } \\
\text { - Diagnosis confirmed on core needle, vacuum-assisted biopsy or } \\
\text { - } \text { surgery } \leq 120 \text { days of registration } \\
\text { - HER }(+) \text { and/or PR(+) by IHC ( } \geq 10 \% \text { staining or Allred score } \geq 4) \\
\text { Histology slides reviewed and agreement between two clinical } \\
\text { pathologists that pathology fulfils COMET eligibility criteria. } \\
\text { At least two sites of biopsy for those cases where mammographic } \\
\text { extent of calcifications exceeds } 4 \mathrm{~cm} \text {, with second biopsy benign or } \\
\text { both sites fulfilling pathology eligibility criteria } \\
\text { Amenable to follow-up examinations } \\
\text { Ability to read, understand and evaluate study materials and } \\
\text { willingness to sign a written informed consent document in Spanish } \\
\text { or English }\end{array}$ & $\begin{array}{l}\text { All grade III DCIS } \\
\text { Male DCIS } \\
\text { Concurrent diagnosis of invasive or microinvasive breast cancer } \\
\text { in either breast prior to randomisation } \\
\text { Documented mass on examination or imaging at the site of DCIS } \\
\text { prior to biopsy yielding diagnosis of DCIS } \\
\text { Bloody nipple discharge or skin changes associated with DCIS } \\
\text { Mammographic finding of BI-RADS } 4 \text { or greater within } 6 \text { months } \\
\text { of registration at site other than that of known DCIS, without } \\
\text { pathological assessment } \\
\text { Use of investigational cancer agents within } 6 \text { weeks prior to } \\
\text { diagnosis } \\
\text { Any serious and/or unstable pre-existing medical, psychiatric or } \\
\text { other existing condition that would prevent compliance with the } \\
\text { trial or consent process } \\
\text { Pregnancy } \\
\text { Documented history of prior tamoxifen, aromatase inhibitor, or } \\
\text { raloxifene in last } 6 \text { months }\end{array}$ \\
\hline
\end{tabular}

$\mathrm{ADH}$, atypical ductal hyperplasia; BI-RADS, Breast Imaging Reporting and Data System; COMET, Comparison of Operative versus Monitoring and Endocrine Therapy; DCIS, ductal carcinoma in situ; ECOG, Eastern Cooperative Oncology Group; ER(+), oestrogen receptor positive; HER2, human epidermal growth factor 2; IHC, immunohistochemistry; PR(+), progesterone receptor positive.

\section{Active surveillance}

Patients in the AS arm will not undergo surgery unless a biopsy during surveillance documents invasive breast disease which requires surgical intervention. If the patient opts for surgery in the absence of invasive breast cancer, they will be considered as a 'crossover' and will continue to participate in completion of patient-reported outcome surveys. Figure 2 presents the surveillance protocol for patients on the AS arm of the study.

\section{Endocrine therapy}

The use of endocrine therapy is not mandatory, but patients are encouraged to discuss this with their providers in both arms of the trial. Selection of endocrine therapy will be determined based on provider recommendation and patient preference, and administered for a maximum duration of 5 years. If applicable, data regarding the use of endocrine therapy (type, duration, adherence and side effects) will be captured at each visit and patient-reported adherence will be measured in follow-up surveys.

All additional follow-up and monitoring beyond that required per protocol (described below) will be conducted according to the standard of care from each provider and institution. The provider will also exercise their best clinical judgement regarding the necessity for baseline laboratory testing (eg, liver function tests, triglycerides) and imaging (eg, breast MRI, dual-energy X-ray absorptiometry scanning).

\section{SURVEILLANCE PROTOCOL}

For both the GCC and AS groups, required surveillance consists of clinical examination, including history and physical examination, every 6 months for a minimum of 5 years and every 12 months thereafter, up to 7 years from the time of registration. Patients on the GCC arm who have not had a mastectomy will have bilateral mammography annually; those on the AS arm will have ipsilateral mammography every 6 months and contralateral mammography every 12 months (table 2 ).

Clinical criteria requiring further investigation include: new breast signs and symptoms such as new breast mass; nipple/skin retraction; nipple discharge and breast oedema/erythema on clinical examination in either breast. Radiographic criteria for biopsy include an increase in extent of calcifications $\geq 5 \mathrm{~mm}$ in at least one dimension compared with the most recent prior MMG in the index breast as well as new suspicious findings on other radiological studies (US, MRI) in either breast (box 1). 


\section{Observation:}

- Clinical breast examination q6 months

- MMG of affected breast

- q6 months for AS

- q12 months for GCC

- MMG of unaffected breast q12 months

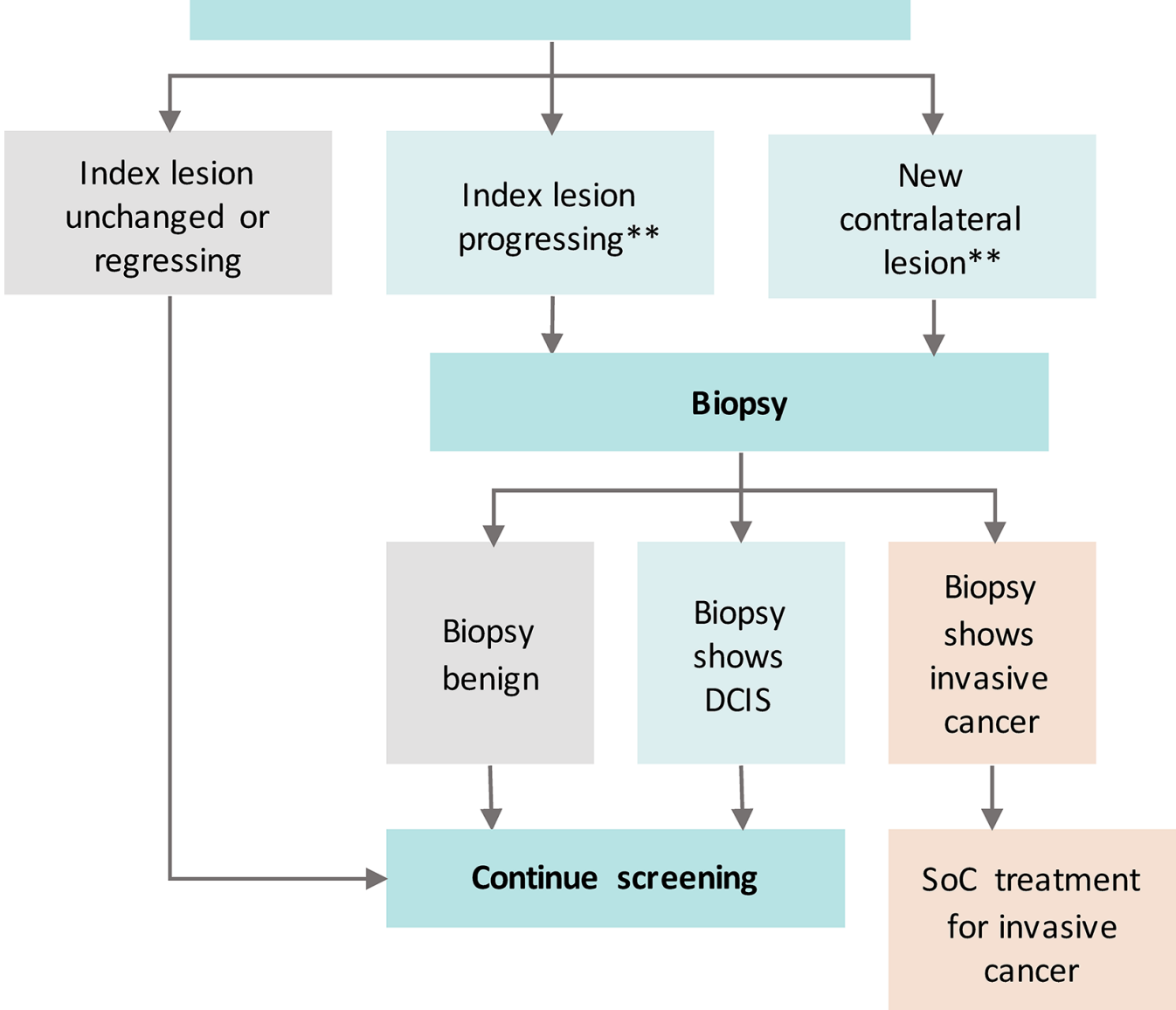

Figure 2 Surveillance protocol for COMET trial. Mammogram (MMG) not required if mastectomy performed. ${ }^{*}$ Criteria for progression: (A) New mass/architectural distortion/density on surveillance MMG in either breast according to American College of Radiology Breast Imaging Reporting and Data System; (B) Increase in extent of calcifications $\geq 5 \mathrm{~mm}$ in at least one dimension compared with the most recent prior MMG of the index breast; (C) New suspicious findings on other radiological studies (ultrasound, MRI) in either breast. AS, active surveillance; COMET, Comparison of Operative versus Monitoring and Endocrine Therapy; DCIS, ductal carcinoma in situ; GCC, guideline concordant care; SoC, standard of care.

\section{Duration of follow-Up}

Progression, recurrence, new primary disease, residual DCIS (or an additional DCIS lesion) and mortality status will be collected up to 10 years from randomisation.

\section{OUTCOMES}

Endpoints were selected in two broad categories: (1) Clinical outcomes defined as those disease-related and treatment-related outcomes to be collected by research staff from primary source documentation and (2) Patient reported outcomes (PRO) which include an array of relevant quality of life and psychosocial outcomes collected from patient surveys (table 3 ).

\section{Primary clinical outcomes}

Ipsilateral breast events

Investigational biopsies will be performed in both study arms for suspicion of a new DCIS or invasive breast cancer in the ipsilateral breast as deemed clinically appropriate by the patient's treatment team. The resulting pathology slides from the biopsy will be reviewed by two pathologists and disease management recommended according to the histological diagnosis. In the GCC arm, any diagnosis will be managed according to standard of care for a local breast event or benign biopsy. In the AS arm, only an invasive breast cancer diagnosis will prompt intervention, according to standard management options. 
Table 2 Schedule of eligibility screening and clinical follow-up

\begin{tabular}{|c|c|c|c|c|c|c|c|}
\hline & & $\begin{array}{l}\text { Eligibility } \\
\text { screening }\end{array}$ & & $\begin{array}{l}\text { Days } \\
1-180\end{array}$ & $\begin{array}{l}\text { Every } \\
6 \text { months } \\
\text { through } \\
\text { year } 5\end{array}$ & $\begin{array}{l}\text { Every } \\
12 \text { months } \\
\text { through } \\
\text { year } 5\end{array}$ & $\begin{array}{l}\text { Every } \\
12 \text { months } \\
\text { through } \\
\text { years 5-7 }\end{array}$ \\
\hline \multirow{4}{*}{$\begin{array}{l}\text { Tests and } \\
\text { observations }\end{array}$} & History and physical & $x$ & & & $x$ & & $x$ \\
\hline & Weight, height & $x$ & & & & & \\
\hline & Pulse, blood pressure & $x$ & & & & & \\
\hline & Randomisation & & & $x$ & & & \\
\hline \multirow{3}{*}{$\begin{array}{l}\text { Imaging } \\
\text { studies }^{\beta}\end{array}$} & Contralateral MMG & & & & & $x$ & \\
\hline & Ipsilateral breast imaging & $X^{\gamma}$ & 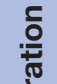 & & $\begin{array}{l}\text { X } \\
\text { (AS only) }\end{array}$ & $\begin{array}{l}\text { X } \\
\text { (GCC only) }\end{array}$ & \\
\hline & Bilateral MMG & $X^{\gamma}$ & $\frac{\omega t}{\pi}$ & & & & $x$ \\
\hline \multirow[t]{3}{*}{$\begin{array}{l}\text { Therapeutic } \\
\text { procedures }\end{array}$} & Surgery & & $\underset{\simeq}{\mathscr{\Sigma}}$ & $\begin{array}{l}\text { X } \\
\text { (GCC only) }\end{array}$ & & & \\
\hline & Radiation & & & $\begin{array}{l}\text { X } \\
\text { (GCC only) }\end{array}$ & & & \\
\hline & $\begin{array}{l}\text { Discussion regarding } \\
\text { endocrine therapy. Initiation } \\
\text { if patient opts for treatment }\end{array}$ & & & $\mathrm{X}$ & & & \\
\hline
\end{tabular}

AS, active surveillance; GCC, guideline concordant care; MMG, mammogram.

\section{Contralateral breast events}

For both AS and GCC arms, contralateral findings for suspicion of new DCIS or invasive breast cancer will also be managed as deemed clinically appropriate by the patient's treatment team. The resulting diagnosis will be managed according to best standard practice as determined by provider recommendation and patient preference. If the new contralateral diagnosis is a DCIS that fulfils criteria for the COMET study, the patient can be offered AS or GCC for this diagnosis.

\section{Secondary outcomes}

Additional clinical outcomes which are also relevant to the differences between the GCC and AS groups include further surgical procedures and regional or distant

\section{Box 1 Criteria for potential ductal carcinoma in situ}

\section{progression and indications for biopsy}

\section{Clinical criteria}

- New breast mass on clinical examination in either breast.

- Other new breast signs including nipple/skin retraction, nipple discharge, breast oedema/erythema in either breast.

\section{Radiographic criteria}

- New mass/architectural distortion/density on surveillance mammogram (MMG) in either breast according to American College of Radiology Breast Imaging Reporting and Data System for mammography in assessment of masses and calcifications. ${ }^{31}$

- Increase in extent of calcifications $\geq 5 \mathrm{~mm}$ in at least one dimension compared with the most recent prior MMG in the index breast.

- New suspicious findings on other radiological studies (ultrasound, MRI) in either breast. metastatic breast cancer events. Since DCIS cells remain trapped within the breast duct and therefore have little potential to spread to distant organ sites and cause symptoms or death, few metastatic events are anticipated.

\section{Patient-reported outcomes}

PRO that are potentially important and relevant to women with DCIS will be elicited longitudinally at pre-specified time points during the study (table 4).

Domains including validated measures specific to arm and breast symptoms, body image and decision-making will be collected. To ensure that there is no excessive burden to patients, and to test content flow and clarity, all surveys were piloted by the Patient Leadership Team (PLT) prior to trial initiation. The surveys are provided in print, online or phone interview versions according to patient preference and are also available in Spanish. All PRO data are entered into PRO-CORE, a study-specific survey data collection platform for web-based assessment of PRO, built and managed by the University of North Carolina Patient-Reported Outcomes Core Facility (UNC PRO-Core).

Collaborating sites send MMG files consisting of the last screening and diagnostic MMG studies that immediately predate the diagnostic core/vacuum-assisted biopsy or surgical excision; submission of biospecimens is also a required component of COMET and an integrated part of the consent process (online supplementary appendix 2 ).

\section{STATISTICAL CONSIDERATIONS}

\section{Sample size}

Sample size for this study was estimated using a two-group test of non-inferiority of proportions, with the 2-year invasive 
Table 3 COMET trial primary and secondary endpoints

\begin{tabular}{|c|c|c|}
\hline & Clinical outcomes & $\begin{array}{l}\text { QOL and psychosocial } \\
\text { patient-reported outcomes }\end{array}$ \\
\hline Primary endpoints & $\begin{array}{l}\text { 2years: } \\
\text { Ipsilateral invasive breast cancer rate } \\
\text { 5, } 7 \text { years: } \\
\text { Ipsilateral invasive breast cancer rate }\end{array}$ & \\
\hline Secondary endpoints & $\begin{array}{l}\text { 2years: } \\
\text { Mastectomy/breast conservation rate } \\
\text { Contralateral invasive breast cancer rate } \\
\text { Overall survival and disease-specific survival } \\
\mathbf{5 , 7} \text { years: } \\
\text { Overall survival and disease-specific survival }\end{array}$ & $\begin{array}{l}\text { Baseline, } 6 \text { months, years } \mathbf{1 - 5 :} \\
\text { Health-related QOL } \\
\text { Anxiety and depression } \\
\text { Baseline, } 2 \text { years: } \\
\text { Intolerance of uncertainty } \\
\text { Baseline: } \\
\text { Coping }\end{array}$ \\
\hline Other endpoints & $\begin{array}{l}\text { 6-12 months: } \\
\text { Number of radiological studies } \\
\text { Number of biopsies } \\
\text { Number of procedures } \\
\text { Rate of crossover and drop-out } \\
\mathbf{2 y e a r s :} \\
\text { Breast MRI rate } \\
\text { Breast biopsy rate } \\
\text { Radiation rate } \\
\text { Chemotherapy rate } \\
\mathbf{5 , 7} \text { years: } \\
\text { Radiation rate } \\
\text { Chemotherapy rate }\end{array}$ & $\begin{array}{l}\text { Baseline, } 6 \text { months, years } 1-5: \\
\text { Pain and other symptoms } \\
\text { Body image and sexual function } \\
\text { Employment status } \\
\text { Self-reported comorbidity } \\
6 \text { months, years 1-5: } \\
\text { Adherence to hormonal therapy } \\
6 \text { months, } 2 \text { years: } \\
\text { Health behaviour/lifestyle factors } \\
\text { Use of complementary therapies } \\
\text { Years 1-5: } \\
\text { Decisional regret } \\
\text { Baseline, } 2 \text { years: } \\
\text { Quality of decision-making } \\
\text { Knowledge and risk perception } \\
6 \text { months: } \\
\text { Financial burden } \\
\text { Baseline: } \\
\text { Decisional conflict }\end{array}$ \\
\hline
\end{tabular}

COMET, Comparison of Operative versus Monitoring and Endocrine Therapy; QOL, quality of life.

breast cancer rate in the GCC group assumed to be 0.10 based on published studies. ${ }^{18} 19$ The non-inferiority margin assumed was 0.05 as this was thought to be a clinically meaningful difference between the two arms, beyond which AS could not be reasonably considered to be equivalent to GCC. Based on a one-sided unpooled z-test, with alpha $=0.05$, a sample size of $n=446$ per group will have $80 \%$ power $^{20}$ to detect the specified non-inferiority margin. A secondary time-to-event analysis will also be performed.

\section{Planned analysis for clinical outcome data}

The primary analysis will not simply follow the intent-to-treat principle but will analyse as randomised all patients with outcomes measured. However, we believe that this trial, as in other studies which randomise to operative versus non-operative arms, will have both non-compliers and contamination, due to patients who will have a desire to avoid surgery as well as patients who, conversely, will have a desire to have any pre-cancerous lesion removed. Thus, the final study design will include a per-protocol component as well as a pragmatic component for those patients who are randomised and decline participation in the assigned arm. We will define a crossover from AS to GCC as any breast surgery on the affected breast in the absence of invasive breast cancer when randomised to AS. Similarly, a crossover from GCC to AS occurs if the patient refuses surgery when randomised to GCC.

\section{Planned subgroup analyses}

Although endocrine therapy is not required on either study arm, we will collect data on its use to determine whether it impacts rates of invasive breast cancer in either group. ${ }^{21}$ Thus, a planned subset analysis of endocrine therapy use will be completed using multivariable logistic regression, with similar adjustments for drop-out and non-adherence. Additionally, factors that may impact the selection of endocrine therapy in both arms such as age and pathological features will also be included. Similarly, we are interested in understanding how imaging modality used may impact assessment of invasive breast cancer during AS, that is, whether MRI detects higher rates of invasive breast cancer than MMG for those patients whose providers opt to include MRI for surveillance. This will also be assessed in the AS group with logistic regression, controlling for factors that could impact selection of MMG versus MRI, such as patient age or breast density. 


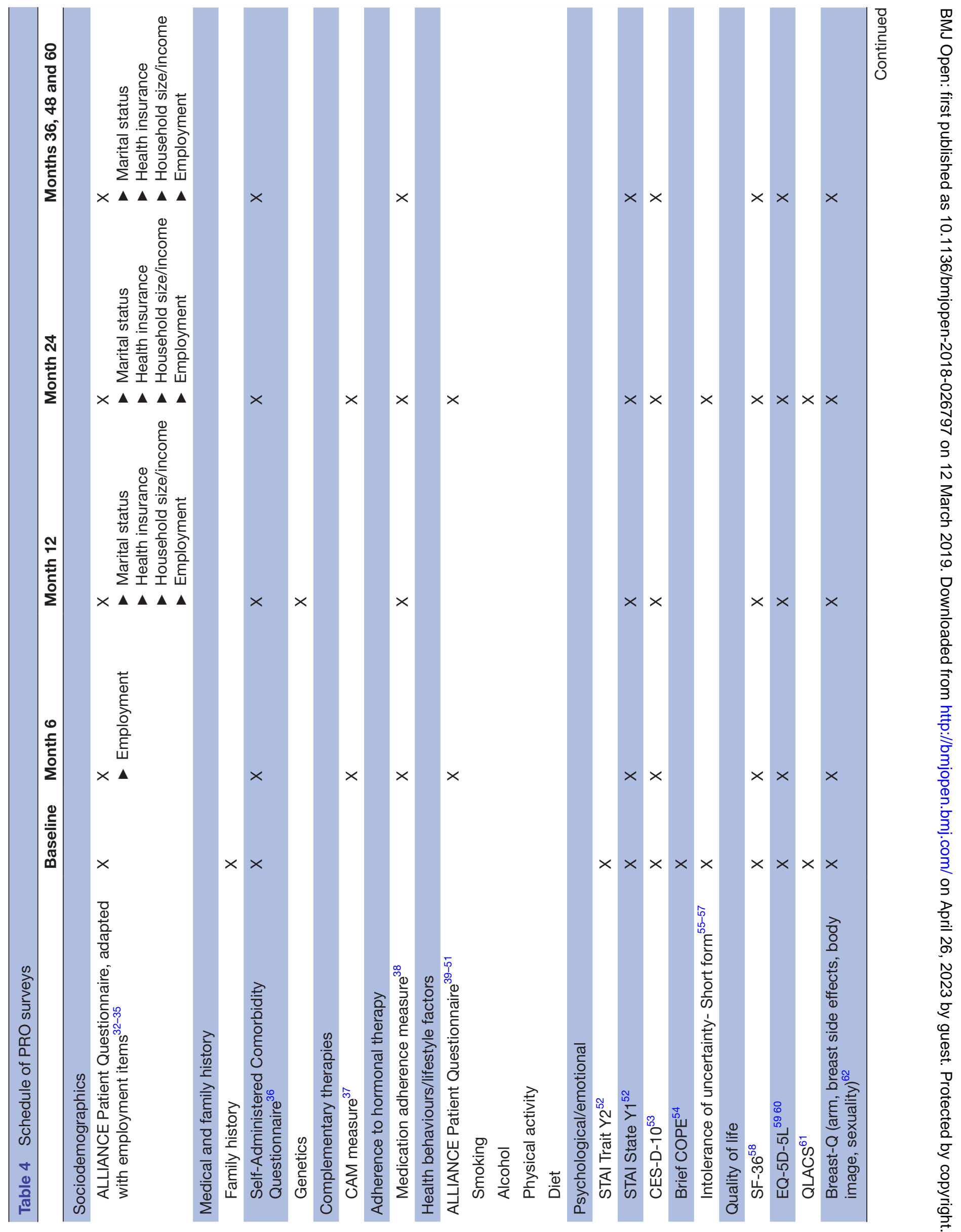




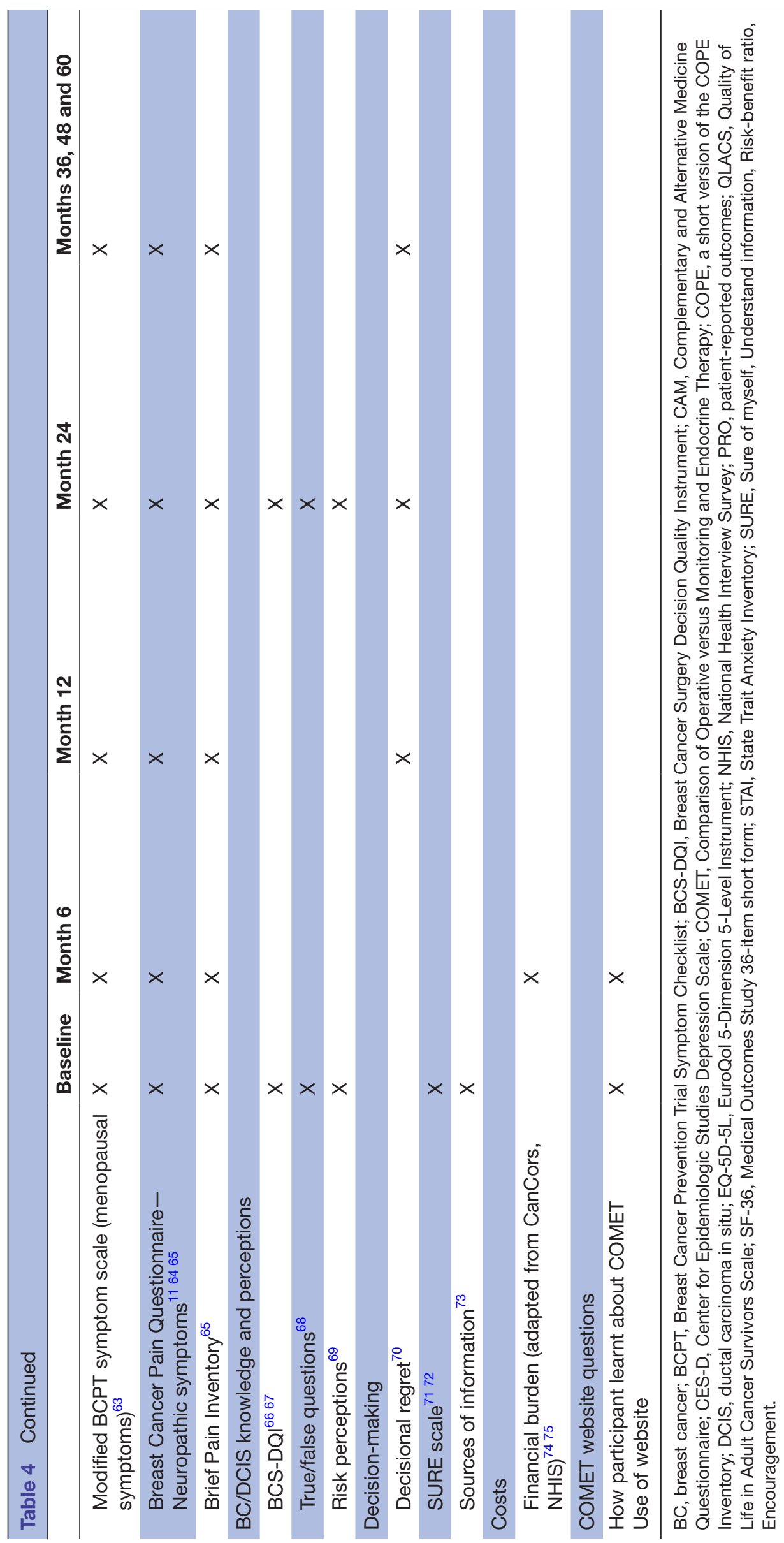


We will also consider menopausal status and baseline risk of breast cancer, and whether these factors influence rate of invasive breast cancer at 2 years. While the study is not powered on these endpoints, these factors are likely to impact outcomes, and thus will be evaluated in planned subset analyses.

\section{PATIENT AND PUBLIC INVOLVEMENT}

Patients, patient advocates and other stakeholders have been actively engaged in the development of this proposal, including the preliminary studies conducted to inform the work. In order to facilitate advocate engagement, we established the PLT. The input and activities of the PLT and advocacy networks guide direction of the trial throughout its entirety.

The PLT have been heavily engaged in the conception of the study (including the original research question) and have partnered in all phases of planning. The PLT collaborated with investigators in the definition of study comparators and outcomes, key constructs to be measured and choice of validated measures to assess those key constructs. The identification of outcomes that the DCIS population of interest notice and care about is particularly relevant in order to provide practical information that can help patients make informed decisions about their health and healthcare. The appropriateness/relevance of survey measures has been reviewed by patient advocates and survey questions have been piloted with them for usability testing.

Patient advocates on the study have diverse involvement or leadership in breast cancer patient advocacy organisations. They have also been strong leaders in the DCIS advocacy community for decades and have deep ties to their constituencies; this will enable them to mentor members of these constituencies who lack this background in order to facilitate their full participation. This active engagement with key stakeholders will be crucial in the compilation of future dissemination strategies/translation of study findings to both professional and patient/public constituencies.

In sum, the PLT: (1) provide input to create effective protocols and survey designs that answer relevant questions for patients, clinicians and research, (2) contribute to the development of educational and implementation tools for clinical sites and patients with DCIS, (3) recruit patient advocates to 'beta' test surveys and patient tools, (4) develop and implement strategies to measure the impact of patient involvement on the advancement of engagement science and (5) monitor accrual and participate in the overall implementation of the study.

\section{ETHICS AND DISSEMINATION}

The COMET trial will be subject to bi-annual formal review at the Alliance Foundation Data Safety Monitoring Board (DSMB) meetings. At each meeting, the
DSMB will review a report of primary and secondary objectives, study schema, definition of primary endpoints, a brief administration summary of current study status, accrual goals versus actual accrual, a summary of patient characteristics to date, a summary of drop-out or crossover from allocated arm, an assessment of data completeness for the various types of data collected, a summary of primary and secondary outcomes by study arm and finally, a summary of adverse events and serious adverse events that will be reported from study entry until 7 years after registration.

\section{Data safety monitoring at interim analysis}

Interim analyses for futility/safety will be completed annually, with reporting following Consolidated Standards of Reporting Trials (CONSORT) guidelines for non-inferiority trials. ${ }^{22}$ We consider stopping under two scenarios: if there is sufficient indication of (1) lack of efficacy or (2) potential harm.

First, we will determine if the deviation of the point estimate of the invasive breast cancer event rate difference for AS versus GCC exceeds 0 by more than 4 SDs or $3 \mathrm{SDs}$ at either of the interim analyses completed when $1 / 2$ and $3 / 4$ of the expected number of total events has accumulated, respectively. The SD will be computed from the Kaplan-Meier estimates at 2 years. In the case that SD exceeds the predetermined bounds, the probability that AS is non-inferior is minimal, and the trial will be halted due to the lack of efficacy.

Second, the trial was developed based on the premise that the upstaging rate to invasive breast cancer is approximately $10 \% .{ }^{23}$ If this rate is substantially higher than $10 \%$, then we would potentially be exposing AS patients to harm. Thus, if the estimate for the upstaging rate in the GCC arm is significantly greater than $10 \%$ based on the Kaplan Meier estimate at 2 years, the trial will be halted due to potential patient harm.

\section{DISCUSSION}

Overdiagnosis and overtreatment may be unintended consequences of mammographic screening. ${ }^{24}$ Given that DCIS is a non-obligate precursor of invasive breast cancer, for those women whose DCIS might never progress even without treatment or whose treatment and outcomes may not differ even if invasion occurs, there is a pressing need to study more selective clinical strategies than the current, non-risk-based therapies for DCIS originally intended for invasive breast cancer. For DCIS at low-risk of progression such as low-grade, small, non-palpable lesions, there may be no significant benefit to surgery or radiation and a de-escalation approach should be tested as it has been in other cancers (eg, prostate cancer). ${ }^{25}{ }^{26}$ There is recognition that high-grade DCIS is more likely to progress to an invasive breast cancer and these patients are excluded from the study. Given the lead-time between the development of DCIS and appearance of invasive breast 
cancer, ${ }^{21}$ there may also be a case for tailoring intervention by age and presence of competing comorbidities.

\section{Global collaboration}

Adoption of significant practice changes in breast cancer treatment has often required consideration of multiple sources of information. Thus, compatibility with other trials is an important goal for the implementation of findings from the COMET study. The LOw Risk dcIS study (LORIS trial; ISRCTN 27544579) 2728 is a randomised controlled trial of AS versus GCC in the UK, which opened to accrual in 2015. The patient populations, healthcare environments and the clinical trials organisation of the COMET and LORIS studies represent an exceptional opportunity to combine resources and strategies, to compare outcomes and to identify similarities and differences in DCIS diagnosis, treatment and surveillance policies both from a patient population and a healthcare systems perspective. To that end, LORIS principal investigators have worked with the COMET team in order to closely align the two studies and allow future meta-analysis of both clinical and PRO endpoints. Specifically, we have prospectively designed the eligibility criteria, outcomes and surveillance protocol which, while not identical in every instance, will nevertheless allow for a planned meta-analysis at completion of both studies. In addition, there is a randomised, international, multi-centre, phase III non-inferiority trial being conducted in the Netherlands (The LORD-LOw Risk DCIS study) ${ }^{29}$ as well as other global efforts to identify biological components of DCIS 'risk'; for example, the Prevent Ductal Carcinoma In Situ Invasive Overtreatment Now (PRECISION) study. ${ }^{30}$

The broad, long-term objective of this proposal is to provide high-quality evidence regarding outcomes of treatment versus surveillance for DCIS and to determine whether data support the inclusion of AS in treatment guidelines for DCIS. It is anticipated that the evidence provided by the COMET study, together with data collected from the other low-risk DCIS studies, will enable patients and stakeholders to make better informed decisions about potential management options for low-risk DCIS.

The COMET study represents an important opportunity to address a highly relevant healthcare issue with broad-reaching health, social and economic implications. Moreover, we hope that this study may provide a framework for evidence development in other low-risk conditions where overtreatment is an emerging concern.

\author{
Author affiliations \\ ${ }^{1}$ Department of Surgery, Division of Surgical Oncology, Duke University, Durham, \\ North Carolina, USA \\ ${ }^{2}$ Department of Biostatistics and Bioinformatics, Duke University, Durham, North \\ Carolina, USA \\ ${ }^{3}$ Patient Leadership Team, COMET Study
}

${ }^{4}$ Department of Health Policy and Management, University of North Carolina, Chapel Hill, North Carolina, USA

${ }^{5}$ Department of Medicine, University of California, San Francisco, California, USA ${ }^{6}$ Department of Medicine, Brigham and Women's Hospital, Dana Farber Cancer Institute, Boston, Massachusetts, USA

${ }^{7}$ Department of Breast Surgery, Division of Surgical Oncology, Baylor College of Medicine, Houston, Texas, USA

${ }^{8}$ Alliance Foundation Trials, Boston, Massachusetts, USA

Acknowledgements We gratefully acknowledge all patients and families who are participating in DCIS clinical trials worldwide whose commitment allow for important advances in DCIS.

Contributors The PI and first author of this paper (ESH) was instrumental in the compilation of this study protocol. Each co-author (TH, TL, EF, DP, DB, DC, AB, CK, $\mathrm{SR}, A T, A W, A P)$ contributed equally to subsequent development of the protocol. EF, DP, DB and DC form the COMET Study Patient Leadership Team.

Funding This work was supported through a Patient-Centered Outcomes Research Institute (PCORI) Award (PCS-1505-30497). The sponsor of the COMET study is Alliance Foundation Trials (https://alliancefoundationtrials.org). Correlative studies are supported by the Breast Cancer Research Foundation (BCRF-17-173). ESH is supported by a Duke Comprehensive Cancer Center Grant (5P30CA014236-44). Patient-reported outcome data are collected via the University of North Carolina Patient Reported Outcomes Core, which is supported in part by grants from the National Institute of Health (DK056350) to the University of North Carolina Nutrition Obesity Research Center, and the US National Cancer Institute (P30 CA016086) to the Lineberger Comprehensive Cancer Center.

Disclaimer All statements in this article are solely those of the authors and do not necessarily represent the views of PCORI, its Board of Governors or Methodology Committee.

\section{Competing interests None declared.}

Patient consent for publication Not required.

Ethics approval Quorum Centralised Institutional Review Board.

Provenance and peer review Not commissioned; peer reviewed for ethical and funding approval prior to submission.

Open access This is an open access article distributed in accordance with the Creative Commons Attribution Non Commercial (CC BY-NC 4.0) license, which permits others to distribute, remix, adapt, build upon this work non-commercially, and license their derivative works on different terms, provided the original work is properly cited, appropriate credit is given, any changes made indicated, and the use is non-commercial. See: http://creativecommons.org/licenses/by-nc/4.0/.

\section{REFERENCES}

1. Ernster VL, Ballard-Barbash R, Barlow WE, et al. Detection of ductal carcinoma in situ in women undergoing screening mammography. $J$ Natl Cancer Inst 2002;94:1546-54.

2. Cancer Facts and Figures. Special section: breast carcinoma in situ. https://www.cancer.org/content/dam/cancer-org/research/cancerfacts-and-statistics/annual-cancer-facts-and-figures/2015/specialsection-breast-carcinoma-in-situ-cancer-facts-and-figures-2015.pdf (Accessed 15 May 2018).

3. Erbas B, Provenzano E, Armes J, et al. The natural history of ductal carcinoma in situ of the breast: a review. Breast Cancer Res Treat 2006;97:135-44.

4. Ozanne EM, Shieh Y, Barnes J, et al. Characterizing the impact of 25 years of DCIS treatment. Breast Cancer Res Treat 2011;129:165-73.

5. Welch HG, Black WC. Overdiagnosis in cancer. J Natl Cancer Inst 2010;102:605-13.

6. Marmot MG, Altman DG, Cameron DA, et al. The benefits and harms of breast cancer screening: an independent review. Br J Cancer 2013;108:2205-40.

7. NCCN. National Comprehensive Cancer Network Announces Abramson Cancer Center of the University of Pennsylvania as New Member Institution. http://www.nccn.org/professionals/physician_ gls/f_guidelines.asp (Accessed 5 May 2018).

8. Worni M, Greenup R, Akushevich I, et al. Trends in treatment patterns and outcomes for DCIS: a SEER population-based analysis. J Clin Oncol ASCO 2014 Meeting Abstracts 2014.

9. Wallis MG, Clements K, Kearins O, et al. The effect of DCIS grade on rate, type and time to recurrence after 15 years of follow-up of screen-detected DCIS. Br J Cancer 2012;106:1611-7. 
10. Andersen KG, Kehlet H. Persistent pain after breast cancer treatment: a critical review of risk factors and strategies for prevention. J Pain 2011;12:725-46.

11. Schreiber KL, Martel MO, Shnol H, et al. Persistent pain in postmastectomy patients: comparison of psychophysical, medical, surgical, and psychosocial characteristics between patients with and without pain. Pain 2013;154:660-8.

12. Bruce J, Thornton AJ, Powell R, et al. Psychological, surgical, and sociodemographic predictors of pain outcomes after breast cancer surgery: a population-based cohort study. Pain 2014;155:232-43.

13. Bruce J, Thornton AJ, Scott NW, et al. Chronic preoperative pain and psychological robustness predict acute postoperative pain outcomes after surgery for breast cancer. Br J Cancer 2012;107:937-46.

14. Worni M, Akushevich I, Greenup R, et al. Trends in treatment patterns and outcomes for ductal carcinoma in situ. J Natl Cancer Inst 2015;107:djv263

15. Dunn G, Maracy M, Tomenson B. Estimating treatment effects from randomized clinical trials with noncompliance and loss to follow-up: the role of instrumental variable methods. Stat Methods Med Res 2005;14:369-95.

16. Kim MY. Using the instrumental variables estimator to analyze noninferiority trials with noncompliance. J Biopharm Stat 2010;20:745-58.

17. Cuzick J, Edwards R, Segnan N. Adjusting for non-compliance and contamination in randomized clinical trials. Stat Med 1997;16:1017-29.

18. Kurniawan ED, Rose A, Mou A, et al. Risk factors for invasive breast cancer when core needle biopsy shows ductal carcinoma in situ. Arch Surg 2010;145:1098-104.

19. Soumian S, Verghese ET, Booth M, et al. Concordance between vacuum assisted biopsy and postoperative histology: implications for the proposed Low Risk DCIS Trial (LORIS). Eur J Surg Oncol 2013;39:1337-40.

20. Chow S, Shao J, Wang H. Sample size calculations in clinical research. London: Chapman \& Hall/CRC Biostatistics Series, 2008.

21. Maxwell AJ, Clements K, Hilton B, et al. Risk factors for the development of invasive cancer in unresected ductal carcinoma in situ. Eur J Surg Oncol 2018;44:429-35.

22. Piaggio G, Elbourne DR, Pocock SJ, et al. Reporting of noninferiority and equivalence randomized trials: extension of the CONSORT 2010 statement. JAMA 2012;308:2594-604.

23. Grimm LJ, Ryser MD, Partridge $A H$, et al. Surgical upstaging rates for vacuum assisted biopsy proven dcis: implications for active surveillance trials. Ann Surg Oncol 2017;24:3534-40.

24. Welch HG. Black WC: Overdiagnosis in cancer. J Natl Cancer Inst 2010;102:605-13.

25. Hamdy FC, Donovan JL, Lane JA, et al. 10-Year outcomes after monitoring, surgery, or radiotherapy for localized prostate cancer. $N$ Engl J Med 2016;375:1415-24.

26. Leapman MS, Cowan JE, Nguyen HG, et al. Active Surveillance in Younger Men With Prostate Cancer. J Clin Oncol 2017;35:1898-904.

27. Francis A, Fallowfield L, Rea D. The LORIS Trial: Addressing overtreatment of ductal carcinoma in situ. Clin Oncol 2015;27:6-8.

28. LORIS. A Phase III trial of surgery versus active monitoring for low risk ductal carcinoma in situ (DCIS). https://www.birmingham.ac.uk/ research/activity/mds/trials/crctu/trials/loris/index.aspx (Accessed 5 May 2018).

29. Clinicaltrials.gov. ClinicalTrials.gov is a database of privately and publicly funded clinical studies conducted around the world. https:// clinicaltrials.gov/ct2/show/NCT02492607 (Accessed 15 May 2018).

30. PRECISION. PRECISION (PREvent ductal Carcinoma In Situ Invasive Overtreatment Now). https://www.dcisprecision.org (Accessed 15 May 2018).

31. D'Orsi CJ SE, Mendelson EB, et al. BI-RADS ${ }^{\circ}$ Atlas ACRReston. VA American College of Radiology, 2013.

32. Ward E, Jemal A, Cokkinides V, et al. Cancer disparities by race/ethnicity and socioeconomic status. CA Cancer J Clin 2004;54:78-93.

33. Bach PB, Schrag D, Brawley OW, et al. Survival of blacks and whites after a cancer diagnosis. JAMA 2002;287:2106-13.

34. Pollitt RA, Swetter SM, Johnson TM, et al. Examining the pathways linking lower socioeconomic status and advanced melanoma. Cancer 2012;118:4004-13.

35. Blinder VS, Eberle CE, Patil S, et al. Correlates of work status during adjuvant chemo- or radiation therapy for breast cancer. Journal of Clinical Oncology 2016;34:6556.

36. Sangha O, Stucki G, Liang MH, et al. The self-administered comorbidity questionnaire: a new method to assess comorbidity for clinical and health services research. Arthritis Rheum 2003:49:156-63.
37. Burstein HJ, Gelber S, Guadagnoli E, et al. Use of alternative medicine by women with early-stage breast cancer. N Engl J Med Overseas Ed 1999;340:1733-9.

38. Voils $\mathrm{Cl}$, Maciejewski $\mathrm{ML}$, Hoyle $\mathrm{RH}$, et al. Initial validation of a self-report measure of the extent of and reasons for medication nonadherence. Med Care 2012;50:1013-9.

39. Godin G, Shephard RJ. Godin leisure-time exercise questionnaire. Med Sci Sports Exerc 1997;29:S36-38.

40. Meyerhardt JA, Giovannucci EL, Holmes MD, et al. Physical activity and survival after colorectal cancer diagnosis. J Clin Oncol 2006;24:3527-34.

41. Meyerhardt JA, Heseltine D, Niedzwiecki D, et al. Impact of physical activity on cancer recurrence and survival in patients with stage III colon cancer: findings from CALGB 89803. J Clin Oncol 2006;24:3535-41.

42. Beasley JM, Kwan ML, Chen WY, et al. Meeting the physical activity guidelines and survival after breast cancer: findings from the after breast cancer pooling project. Breast Cancer Res Treat 2012;131:637-43.

43. Holmes MD, Chen WY, Feskanich D, et al. Physical activity and survival after breast cancer diagnosis. JAMA 2005;293:2479-86.

44. Peters EN, Torres E, Toll BA, et al. Tobacco assessment in actively accruing national cancer institute cooperative group program clinical trials. J Clin Oncol 2012;30:2869-75.

45. Land SR, Cronin WM, Wickerham DL, et al. Cigarette smoking, obesity, physical activity, and alcohol use as predictors of chemoprevention adherence in the national surgical adjuvant breast and bowel project $\mathrm{p}-1$ breast cancer prevention trial. Cancer Prev Res 2011;4:1393-400.

46. Braithwaite $\mathrm{D}$, Izano $\mathrm{M}$, Moore $\mathrm{DH}$, et al. Smoking and survival after breast cancer diagnosis: a prospective observational study and systematic review. Breast Cancer Res Treat 2012;136:521-33.

47. Ligibel J. Lifestyle factors in cancer survivorship. J Clin Oncol 2012;30:3697-704.

48. Chlebowski RT, Blackburn GL, Thomson CA, et al. Dietary fat reduction and breast cancer outcome: interim efficacy results from the women's intervention nutrition study. J Natl Cancer Inst 2006;98:1767-76.

49. Paxton AE, Strycker LA, Toobert DJ, et al. Starting the conversation performance of a brief dietary assessment and intervention tool for health professionals. Am J Prev Med 2011;40:67-71.

50. Meyerhardt JA, Niedzwiecki D, Hollis D, et al. Association of dietary patterns with cancer recurrence and survival in patients with stage III colon cancer. JAMA 2007;298:754-64.

51. Meyerhardt JA, Sato K, Niedzwiecki D, et al. Dietary glycemic load and cancer recurrence and survival in patients with stage III colon cancer: findings from CALGB 89803. J Natl Cancer Inst 2012:104:1702-11.

52. Marteau TM, Bekker H. The development of a six-item short-form of the state scale of the Spielberger State-Trait Anxiety Inventory (STAI). Br J Clin Psychol 1992;31:301-6.

53. Radloff LS. The CES-D scale: A self-report depression scale for research in the general population. Appl Psychol Meas 1977;1:385-401.

54. Carver CS. You want to measure coping but your protocol's too long: consider the brief COPE. Int J Behav Med 1997;4:92-100.

55. Freeston $\mathrm{MH}$, Rhéaume $\mathrm{J}$, Letarte $\mathrm{H}$, et al. Why do people worry? Pers Individ Dif 1994;17:791-802.

56. Buhr K, Dugas MJ. The Intolerance of uncertainty scale: psychometric properties of the english version. Behav Res Ther 2002;40:931-45

57. Tan HJ, Marks LS, Hoyt MA, et al. The Relationship between Intolerance of Uncertainty and Anxiety in Men on Active Surveillance for Prostate Cancer. J Urol 2016;195:1724-30.

58. Ware JE, Sherbourne CD. The MOS 36-item short-form health survey (SF-36). I. Conceptual framework and item selection. Med Care 1992:30:473-83.

59. Burstein HJ, Gelber S, Guadagnoli E, et al. Use of alternative medicine by women with early-stage breast cancer. $N$ Engl J Med 1999;340:1733-9.

60. Godin G, Shephard RJ. Godin leisure-time exercise questionnaire. Med Sci Sports Exerc 1997;29:S36-8.

61. Avis NE, Smith KW, McGraw S, et al. Assessing quality of life in adult cancer survivors (QLACS). Qual Life Res 2005;14:1007-23.

62. Pusic AL, Klassen AF, Scott AM, et al. Development of a new patientreported outcome measure for breast surgery: the BREAST-Q. Plast Reconstr Surg 2009;124:345-53.

63. Stanton AL, Bernaards CA, Ganz PA. The BCPT symptom scales: a measure of physical symptoms for women diagnosed with or at risk for breast cancer. J Natl Cancer Inst 2005;97:448-56. 
64. Belfer I, Schreiber KL, Shaffer JR, et al. Persistent postmastectomy pain in breast cancer survivors: analysis of clinical, demographic, and psychosocial factors. J Pain 2013;14:1185-95.

65. Gärtner R, Jensen MB, Nielsen J, et al. Prevalence of and factors associated with persistent pain following breast cancer surgery. JAMA 2009;302:1985-92.

66. Sepucha KR, Belkora JK, Chang Y, et al. Measuring decision quality: psychometric evaluation of a new instrument for breast cancer surgery. BMC Med Inform Decis Mak 2012;12:51.

67. Sepucha K, Ozanne E, Silvia K, et al. An approach to measuring the quality of breast cancer decisions. Patient Educ Couns 2007;65:261-9.

68. Bluman LG, Borstelmann NA, Rimer BK, et al. Knowledge, satisfaction, and perceived cancer risk among women diagnosed with ductal carcinoma in situ. J Womens Health Gend Based Med 2001;10:589-98.

69. Lerman C, Croyle R. Psychological issues in genetic testing for breast cancer susceptibility. Arch Intern Med 1994;154:609-16.
70. Brehaut JC, O'Connor AM, Wood TJ, et al. Validation of a decision regret scale. Med Decis Making 2003;23:281-92.

71. O'Connor AM. Validation of a decisional conflict scale. Med Decis Making 1995;15:25-30.

72. Légaré $\mathrm{F}$, Kearing S, Clay K, et al. Are you SURE?: assessing patient decisional conflict with a 4-item screening test. Can Fam Physician 2010;56:e308-14.

73. Rosenberg SM, Tracy MS, Meyer ME, et al. Perceptions, knowledge, and satisfaction with contralateral prophylactic mastectomy among young women with breast cancer: a cross-sectional survey. Ann Intern Med 2013;159:373-81.

74. Kent EE, Forsythe LP, Yabroff KR, et al. Are survivors who report cancer-related financial problems more likely to forgo or delay medical care? Cancer 2013;119:3710-7.

75. van Ryn M, Sanders S, Kahn K, et al. Objective burden, resources, and other stressors among informal cancer caregivers: a hidden quality issue? Psychooncology 2011;20:44-52. 